\title{
Commensurators of finitely generated nonfree Kleinian groups
}

\author{
CHRISTOPHER LEININGER
}

DARREN D LONG

ALAN W REID

\begin{abstract}
We show that any finitely generated torsion-free nonfree Kleinian group of the first kind which is not a lattice and contains no parabolic elements has discrete commensurator.
\end{abstract}

20H10; 20F60, 57M50

\section{Introduction}

Let $G$ be a group and $\Gamma_{1}, \Gamma_{2}<G$. The subgroups $\Gamma_{1}$ and $\Gamma_{2}$ are called commensurable if $\Gamma_{1} \cap \Gamma_{2}$ has finite index in both $\Gamma_{1}$ and $\Gamma_{2}$. The commensurator of a subgroup $\Gamma<G$ is defined to be

$$
C_{G}(\Gamma)=\left\{g \in G: g \Gamma g^{-1} \text { is commensurable with } \Gamma\right\} .
$$

When $G$ is a semisimple Lie group, and $\Gamma$ a lattice, a fundamental dichotomy established by Margulis [26], determines that $C_{G}(\Gamma)$ is dense in $G$ if and only if $\Gamma$ is arithmetic, and moreover, when $\Gamma$ is nonarithmetic, $C_{G}(\Gamma)$ is again a lattice.

Historically, the prominence of the commensurator was due in large part to its density in the arithmetic setting being closely related to the abundance of Hecke operators attached to arithmetic lattices. These operators are fundamental objects in the theory of automorphic forms associated to arithmetic lattices (see Shimura [38] for example). More recently, the commensurator of various classes of groups has come to the fore due to its growing role in geometry, topology and geometric group theory; for example in classifying lattices up to quasi-isometry, classifying graph manifolds up to quasiisometry, and understanding Riemannian metrics admitting many "hidden symmetries" (for more on these and other topics see Bartholdi and Bogopolski [2], Behrstock and Neumann [4], Farb and Weinberger [17; 18], Leininger and Margalit [25], Schwartz [34] and Shalom [37]). 
This article was inspired by a question (apparently first raised by Y Shalom) of whether there is a broad generalization of the theorem of Margulis alluded to above; where one replaces the finite covolume hypothesis by the weaker assumption that the group only be Zariski dense:

Suppose $\Gamma$ is a Zariski-dense discrete subgroup of a semisimple Lie group $G$. If the commensurator of $\Gamma$ is not discrete, must $\Gamma$ be arithmetic (and therefore finite covolume)?

Here we study, and come close to resolving, this question in the first nontrivial case, namely $C_{G}(\Gamma)$ when $G=\operatorname{PSL}(2, \mathbf{C})$ and $\Gamma$ is a finitely generated nonelementary Kleinian group. In this setting, we will abbreviate the notation for the commensurator of $\Gamma$ to $C(\Gamma)$. When $\Gamma$ is of finite covolume and nonarithmetic, identifying $C(\Gamma)$ has attracted considerable attention (see for example Goodman, Heard and Hodgson [19], Neumann and Reid [32] and Reid and Walsh [33]). Our focus here are those Kleinian groups $\Gamma$ for which $\mathbf{H}^{3} / \Gamma$ has infinite volume. Henceforth, unless otherwise stated, the Kleinian groups $\Gamma$ that we consider will always have infinite covolume.

If $\Gamma$ is a Kleinian group, we denote by $\Lambda_{\Gamma}$ and $\Omega_{\Gamma}$ the limit set and domain of discontinuity of $\Gamma$. The Kleinian group $\Gamma$ is said to be of the first kind (resp. second kind) if $\Omega_{\Gamma}=\varnothing$ (resp. $\left.\Omega_{\Gamma} \neq \varnothing\right)$. The only known result for Kleinian groups $\Gamma$ as above is due to L Greenberg [20; 21] who proved the following result (see Section 3.1 for a new proof when there are no parabolics).

Theorem 1.1 Let $\Gamma$ be a finitely generated nonelementary Kleinian group of the second kind and assume that $\Lambda_{\Gamma}$ is not a round circle. Then $[C(\Gamma): \Gamma]<\infty$. In particular, $C(\Gamma)$ is a discrete subgroup of $\operatorname{PSL}(2, \mathbf{C})$.

The exclusion of the limit set being a round circle is to rule out arithmetic Fuchsian subgroups of PSL(2, R), which by Margulis [26] have nondiscrete commensurator. Thus it remains to understand $C(\Gamma)$ when $\Gamma$ is of the first kind. These have been the most difficult Kleinian groups to understand, even in the case that $\Gamma$ is isomorphic to a closed surface group. Indeed, it is only very recently that their geometry has been clarified; see Minsky [28], Brock, Canary and Minsky [7], Mj [30], Agol [1] and Calegari and Gabai [9].

Our main result is:

Theorem 1.2 Let $\Gamma$ be a finitely generated torsion-free Kleinian group of the first kind which is not a lattice. If $\Gamma$ is not free and contains no parabolic elements, then $C(\Gamma)$ is discrete.

Furthermore $[C(\Gamma): \Gamma]=\infty$ if and only if $\Gamma$ is a fibre group. In this case, $C(\Gamma)$ is a lattice. 
We recall that a Kleinian group $\Gamma$ is called a fibre group if there is a finite volume hyperbolic 3-manifold $M=\mathbf{H}^{3} / \Delta$ which fibres over a 1 -orbifold such that $\Gamma<\Delta$ is the subgroup corresponding to the fibre.

The torsion-free assumption is simply to make the statement succinct. Since every finitely generated Kleinian group contains a torsion free subgroup of finite index, it is clear that Theorem 1.2 holds in the presence of torsion with only a mild change of terminology for fibre groups in this setting.

We suspect that Theorem 1.2 holds for any finitely generated Kleinian group which is not a lattice, that is, without the hypothesis on parabolics or freeness. In fact, the proof can be slightly modified to work without the assumption on parabolics, provided we further assume that $\Gamma$ is not the fundamental group of a compression body with toroidal lower boundary; see Section 4. However, there remain issues with generalizing the proof to the case of a free Kleinian group, with or without parabolics.

The proof of Theorem 1.2 makes use of recent progress in understanding finitely generated geometrically infinite Kleinian groups $\Gamma$. In particular, our proof appeals directly to work of $\mathrm{M} \mathrm{Mj}[30 ; 31]$ on the existence of "Cannon-Thurston maps" (see also Section 2 for more discussion on this), which in turn relies on existence of "models for simply degenerate ends" following Minsky [28] and Brock, Canary and Minsky [7;8]. In turn, the complete classification of Kleinian groups via their end invariants also uses the solution to the Tameness Conjecture (by Agol [1] and Calegari and Gabai [9]), although in our case, the application of the Cannon-Thurston map arises for geometrically infinite Kleinian surface groups, and tameness of the quotients of $\mathbf{H}^{3}$ was already established by work of Bonahon [6].

An important ingredient of our proof is of some independent interest. This result is due to M Kapovich [23] in a special case of fiber groups, and the proof we give here is essentially the same as that given by Kapovich. We defer a precise statement to Theorem 3.4, but it can be described informally as follows. The Cannon-Thurston map $\pi: S^{1} \longrightarrow S^{2}$ is obtained via a certain decomposition map; in particular it picks out in the image a collection of exceptional points, namely those points $p \in S^{2}$ for which $\left|\pi^{-1}(p)\right|>1$. The result of Theorem 3.4 is:

Theorem 1.3 The exceptional points of the Cannon-Thurston map are not conical limit points.

By the solution to the Tameness Conjecture [1;9], and the Covering Theorem of Canary [11] (see Theorem 2.1), every finitely generated geometrically infinite subgroup of a uniform lattice in $\operatorname{PSL}(2, \mathbf{C})$ is isomorphic to a closed surface group. Thus a corollary of Theorems 1.1 and 1.2 is the following result. This extends a result of the third author (see Canary [12, Theorem 8.7]). 
Corollary 1.4 Let $\Delta$ be a uniform lattice in $\operatorname{PSL}(2, \mathbf{C})$, and $\Gamma<\Delta$ a finitely generated subgroup of infinite index for which $\Lambda_{\Gamma}$ is not a circle. Then $C(\Gamma)$ is a discrete subgroup of PSL $(2, \mathbf{C})$.

Recently, inspired by this paper, $\mathrm{M} \mathrm{Mj}$ has announced that he has proved Theorem 1.2 in complete generality.

Acknowledgements The authors thank Yehuda Shalom for useful correspondence on the subject of this paper, and Dick Canary, Yair Minsky and Hossein Namazi for helpful conversations.

\section{Ending laminations and Cannon-Thurston maps}

The proof of Theorem 1.2 requires some background about hyperbolic 3-manifolds and from the theory of laminations, ending laminations and Cannon-Thurston maps. We summarize what we need here.

\section{1}

We begin by recalling some basic structure for laminations on a closed surface $S$ (see Casson and Bleiler [15] for further details).

Let $S$ be a closed orientable surface of genus $g \geq 2$ equipped with a fixed complete hyperbolic metric of constant curvature -1 . A (geodesic) lamination $L$ on $S$ is a closed subset foliated by geodesics. A component of $S \backslash L$ is called a principal region for $L$, of which there are only finitely many (see [15, Lemma 4.3]). A lamination $L \subset S$ is filling if it has no proper sublaminations and all principal regions are ideal polygons. In particular $L$ has no closed leaves.

Given a lamination $L \underset{\widetilde{S}}{\subset} S$, we let $\widetilde{L} \subset \underset{\widetilde{S}}{\widetilde{S}}$ denote the preimage of $L$ in the universal cover $\tilde{S}$ of $S$. If $\tilde{\ell}$ (respectively, $\widetilde{P}$ ) is a leaf (respectively, principal region) of $\tilde{L}$, then we write $\partial_{\infty} \tilde{\ell}$ (respectively, $\partial_{\infty} \widetilde{P}$ ) for the intersection of the closure of $\tilde{\ell}$ (respectively, $\widetilde{P}$ ) with $S_{\infty}^{1}$, the circle at infinity of $\widetilde{S}$.

\section{2}

Let $\Gamma$ be a finitely generated nonelementary Kleinian group without parabolics and $N=\mathbf{H}^{3} / \Gamma$. Throughout the paper the convex core of $N$ will be denoted by $C_{N}$; ie $C_{N}=C H\left(\Lambda_{\Gamma}\right) / \Gamma$, where $C H\left(\Lambda_{\Gamma}\right)$ is the convex hull of $\Lambda_{\Gamma}$ in $\mathbf{H}^{3}$. If $C_{N}$ is compact, then $\Gamma$ is said to be geometrically finite (or convex cocompact), otherwise $\Gamma$ 
is called geometrically infinite. We will also write $M \subset N$ for a fixed compact core of $N$. This is a compact submanifold of $N$ for which the inclusion map is a homotopy equivalence (see Scott [35]).

Fix some component $S \subset \partial M$. Let $U \subset(N \backslash M)$ be the component with $S \subset \bar{U}$. This $U$ is a neighborhood of an end $E$ of $N$, and we say that $E$ abuts $S$. This defines a bijection between the set of ends of $N$ and the components of $\partial M$. According to the Tameness Theorem [1; 9], we may arrange that $U \cong S \times(0, \infty)$.

Following Thurston [40], the end $E$ of $N$ abutting $S \subset \partial M$ is called geometrically finite if $E$ contains a neighbourhood $U$ such that $U \cap C_{N}=\varnothing$. The group $\Gamma$ is geometrically finite if and only if all ends of $C_{N}$ are geometrically finite. In this case, we can take $M=C_{N}$.

If $S \subset \partial M$ is incompressible (the only case of interest for us in the following definition), the end $E$ of $N$ abutting $S$ is simply degenerate if there exists a sequence of simple closed curves $\left\{\alpha_{i}\right\}$ in $S$ such that the geodesic representatives $\left\{\alpha_{i}^{*}\right\}$ in $N$ exit the end $E$. The Tameness Theorem implies that every end is either geometrically finite or simply degenerate.

Furthermore, Thurston [39] and Canary [10] showed how to associate to a simply degenerate end $E$, a lamination (the so-called ending lamination of $\mathrm{E}$ ) which is a limit of the simple closed curves $\left\{\alpha_{i}\right\}$ (in an appropriate topology). We shall denote the ending lamination associated to the simply degenerate end $E$ by $v_{E}$. If $v_{E}$ is an ending lamination then it is known to be filling (see Section 8 of Canary [10] for example). For later reference, we observe that the ending lamination is independent of the choice of sequence of simple closed curves and it follows that if one takes a finite sheeted covering $\widetilde{E}$ of an end $E$, the ending lamination of $\widetilde{E}$ is the preimage under the induced covering map of the ending lamination of $E$.

\section{3}

An important tool for us is Canary's Covering Theorem [11] (which extended a result of Thurston [40]; see also Agol [1]). The version we state here can be found in Canary and Leininger [13].

Theorem 2.1 Suppose that $\widehat{N}=\mathbf{H}^{3} / \Gamma$ is a hyperbolic 3-manifold with no cusps and finitely generated fundamental group and $N$ is a hyperbolic 3-orbifold. If $p: \widehat{N} \rightarrow N$ is a cover which is infinite-to-one on a neighborhood $U$ of a simply degenerate end of $\widehat{N}$, then $N$ is closed and has a finite manifold cover $N^{\prime}=\mathbf{H}^{3} / \Gamma^{\prime} \rightarrow N$ such that either 
(1) $N^{\prime}$ fibers over the circle and $\hat{N}$ is the cover associated to a fiber subgroup of $\Gamma^{\prime}$, or

(2) $N^{\prime}$ fibers over the orbifold $S^{1} /\langle z \mapsto \bar{z}\rangle$ and $\hat{N}$ is the cover of $N^{\prime}$ associated to a singular fiber subgroup of $\pi_{1}\left(N^{\prime}\right)$.

In the conclusion of the theorem, the group $\Gamma$ is a fibre group and $N$ is an $\mathbf{R}$-bundle over a surface. In the first case, $N$ is a product, while in the second, it is a twisted R-bundle.

\section{4}

Now suppose that $\Gamma$ is a surface group, so that the compact core is a product $M=$ $S \times[0,1]$, where $S$ is closed orientable surface of genus at least 2 . In this case, there are exactly two ends $E_{+}$and $E_{-}$. If $\Gamma$ is geometrically infinite, one or both of the ends is simply degenerate and we say that $\Gamma$ is singly degenerate or doubly degenerate in these two cases, respectively. For the remainder of this section, we assume that $\Gamma$ is doubly degenerate. For example, $\Gamma$ may be a fibre group of the first type described in the previous subsection. The ending laminations $v_{+}=v_{E_{+}}$and $v_{-}=v_{E_{-}}$can be viewed as laminations on $S$.

If $\Gamma$ contains a finite index subgroup which is the fundamental group of an orientable surface, then we will say that $\Gamma$ is virtually a surface group. It follows from [22, Theorem 10.5] that if $\Gamma$ is virtually a surface group then $N=\mathbf{H}^{3} / \Gamma$ is an $\mathbf{R}$-bundle over a surface, and hence either $\Gamma$ or a canonical index two subgroup $\Gamma_{0}<\Gamma$ is a surface group.

The inclusion map $S \hookrightarrow S \times\{1 / 2\} \subset M \subset N$ induces a representation

$$
\rho: \pi_{1}(S) \rightarrow \Gamma<\operatorname{PSL}(2, \mathbf{C})
$$

and lifts to an equivariant map

$$
\iota: \widetilde{S} \rightarrow \mathbf{H}^{3} \text {. }
$$

The equivariance is with respect to $\rho$ via the action of $\pi_{1}(S)$ on $\widetilde{S}$ by covering transformations and $\Gamma$ on $\mathbf{H}^{3}$

$$
\iota \circ \gamma=\rho(\gamma) \circ \iota \text { for all } \gamma \in \pi_{1}(S) \text {. }
$$

In [14], Cannon and Thurston proved that if $\Gamma$ is a fibre group, then $\iota$ admits a continuous equivariant extension to the compactifications

$$
\widehat{\imath}: \tilde{S} \cup S_{\infty}^{1} \rightarrow \mathbf{H}^{3} \cup S_{\infty}^{2}
$$


The existence of such an extension was subsequently proven by Minsky [27] replacing the fibre assumption with the weaker assumption of bounded geometry (ie a there is a lower bound to the injectivity radius). Existence in the general case was proven more recently by $\mathrm{Mj}$ [30], and $\hat{\imath}$ is referred to as a Cannon-Thurston map for $\Gamma$ or $\rho$. We state Mj's Theorem here together with the description of the map that we will need (see $\mathrm{Mj}$ [31, Theorem 1.3]).

Theorem 2.2 Given a representation $\rho: \pi_{1}(S) \rightarrow \operatorname{PSL}(2, \mathbf{C})$ with doubly degenerate image $\Gamma=\rho\left(\pi_{1}(S)\right)$ as above, there exists a Cannon-Thurston map

$$
\widehat{\iota}: \widetilde{S} \cup S_{\infty}^{1} \rightarrow \mathbf{H}^{3} \cup S_{\infty}^{2}
$$

If $\nu_{ \pm}$are the ending laminations and $a, b \in S_{\infty}^{1}$, then $\hat{\imath}(a)=\widehat{\imath}(b)$ if and only if $a$ and $b$ are either ideal end points of a leaf, or ideal endpoints of a principal region of one of $\tilde{v}_{+}$or $\tilde{v}_{-}$.

It is straightforward to show that if $a$ and $b$ are either ideal end points of a leaf of $\widetilde{v}_{ \pm}$ or ideal endpoints of a principal region of $\widetilde{v}_{ \pm}$then they are identified by the CannonThurston map (see Lemma 3.5 of Mitra [29]). The hard part is to show that this is all that is collapsed.

\section{Proof of Theorem 1.2}

The proof of Theorem 1.2 proceeds by examining two cases. The first is the case where neither $\Gamma$ nor an index two subgroup is a surface group.

\subsection{The case that $\Gamma$ is not virtually a surface group}

We will prove the following result. This implies the aforementioned case of Theorem 1.2 , and in addition gives a new proof Greenberg's result when there are no parabolics (cf Theorem 1.1).

Theorem 3.1 Let $\Gamma$ be a finitely generated torsion-free Kleinian group without parabolic elements for which $\Lambda_{\Gamma}$ is not contained in a round circle. In addition, assume that if $\Gamma$ is of the first kind it is not a lattice or isomorphic to a free group or a surface group.

Then $C(\Gamma)$ is discrete and $[C(\Gamma): \Gamma]<\infty$. 
The hypothesis that $\Lambda_{\Gamma}$ is not contained in a round circle can be replaced by the assumption that $\Lambda_{\Gamma}$ is not equal to a round circle. The proof is a (simpler) twodimensional version of the argument we give here, and we leave the details to the interested reader.

Proof Let $q: \mathbf{H}^{3} \longrightarrow N$ denote the universal covering map.

Suppose first that $\Gamma$ is of the second kind so that $\Omega_{\Gamma} \neq \varnothing$. In this case, there is at least one geometrically finite end of $N=\mathbf{H}^{3} / \Gamma$. Thurston [40] showed that each component of $\partial C_{N}$ is a pleated surface (see also Epstein and Marden [16]) which we may assume is also a component of $\partial M$. For each geometrically finite end $E$ of $N$, we let $\mathcal{P}_{E}$ denote this pleated surface which $E$ abuts. Let $E_{1}, \ldots, E_{k}$ denote the geometrically finite ends of $N$ and write

$$
\mathcal{P}_{N}=\left\{\mathcal{P}_{E_{1}}, \ldots, \mathcal{P}_{E_{k}}\right\}
$$

The preimage of this finite set of pleated surfaces in $\mathbf{H}^{3}$ is a locally finite collection of connected pleated surfaces in $\mathbf{H}^{3}$

$$
\mathcal{P}_{\Gamma}=q^{-1}\left(\mathcal{P}_{N}\right)
$$

If $\widehat{\Gamma}<\Gamma$ is a finite index subgroup then

$$
\mathcal{P}_{\Gamma}=\mathcal{P}_{\widehat{\Gamma}} .
$$

To see this, first observe that compact cores and convex cores are natural with respect to finite coverings. Therefore every neighborhood of an end of $\hat{N}$ finitely covers the neighborhood of an end of $N$ via the associated covering $p: \widehat{N} \rightarrow N$, preserving geometric finiteness. If $\widehat{E}_{1}, \ldots, \widehat{E}_{r}$ are the ends of $\widehat{N}$ with neighborhoods covering a neighborhood of $E$, we have

$$
p^{-1}\left(\mathcal{P}_{E}\right)=\left\{\mathcal{P}_{\widehat{E}_{1}}, \ldots, \mathcal{P}_{\widehat{E}_{r}}\right\} .
$$

From this it follows that $p^{-1}\left(\mathcal{P}_{N}\right)=\mathcal{P}_{\widehat{N}}$, and consequently (1) holds.

Given $\gamma \in \operatorname{PSL}(2, \mathbf{C})$ observe that

$$
\mathcal{P}_{\gamma \Gamma \gamma^{-1}}=\gamma\left(\mathcal{P}_{\Gamma}\right)
$$

Furthermore, if $\gamma \in C(\Gamma), \hat{\Gamma}=\gamma \Gamma \gamma^{-1} \cap \Gamma$ has finite index in both $\Gamma$ and $\gamma \Gamma \gamma^{-1}$, so combining (1) and (2), we have

$$
\gamma\left(\mathcal{P}_{\Gamma}\right)=\mathcal{P}_{\Gamma}
$$

Therefore, $\quad C(\Gamma)<\operatorname{Stab}\left(\mathcal{P}_{\Gamma}\right)=\left\{g \in \operatorname{PSL}(2, \mathbf{C}) \mid g \mathcal{P}_{\Gamma}=\mathcal{P}_{\Gamma}\right\}$,

and so discreteness of $C(\Gamma)$ follows from the next claim. 


\section{Claim $\operatorname{Stab}\left(\mathcal{P}_{\Gamma}\right)$ is discrete.}

Proof of Claim Each surface in $\mathcal{P}_{\Gamma}$, contains countably many flat pieces, each of which is contained in a hyperbolic plane $\mathbf{H}^{2} \subset \mathbf{H}^{3}$. Let $\left\{\mathbf{H}_{i}^{2}\right\}$ be the set of all such hyperbolic planes with $\mathbf{H}_{i}^{2}$ containing the flat piece $F_{i}$ of some pleated surface in $\mathcal{P}_{\Gamma}$. The intersection $\bigcap_{i} \mathbf{H}_{i}^{2}$ is invariant by $\operatorname{Stab}\left(\mathcal{P}_{\Gamma}\right)$ (and so also $\Gamma$ ), and since $\Lambda_{\Gamma}$ is not contained in a round circle, it follows that this intersection is empty. Therefore, there are a finite set of flat pieces, say $F_{1}, \ldots, F_{k}$, of surfaces in $\mathcal{P}_{\Gamma}$ so that the associated hyperbolic planes intersect trivially

$$
\mathbf{H}_{1}^{2} \cap \cdots \cap \mathbf{H}_{k}^{2}=\varnothing .
$$

Now suppose that $\left\{\gamma_{n}\right\}_{n=1}^{\infty} \subset \operatorname{Stab}\left(\mathcal{P}_{\Gamma}\right)$ is a sequence converging to the identity. Since $\mathcal{P}_{\Gamma}$ is locally finite, after passing to a subsequence we can assume that each $F_{i}$ is invariant by $\gamma_{n}$ for $i=1, \ldots, k$ and all $n$. Therefore, $\mathbf{H}_{i}^{2}$ is invariant by $\gamma_{n}$ for each $i=1, \ldots, k$ and all $n$. Since $\mathbf{H}_{1}^{2} \cap \cdots \cap \mathbf{H}_{k}^{2}=\varnothing$ it follows that $\gamma_{n}$ is the identity, and hence $\operatorname{Stab}\left(\mathcal{P}_{\Gamma}\right)$ is discrete.

We now assume that $\Gamma$ is of the first kind. The idea of the proof is similar to the previous case: to each end $E$ of $N$ we associate a finite, nonempty set of pleated surfaces $\mathcal{P}_{E}$ in $N$. This will be done so that the preimage $\mathcal{P}_{\Gamma}=q^{-1}\left(\mathcal{P}_{N}\right)$ satisfies (1) and (2). The proof can then be completed exactly as above.

Given an end $E \subset N$, let $S \subset \partial M$ be the component which it abuts. According to Bonahon [5, Theorem 2.1] there is a unique (up to isotopy) compression body $B_{S} \subset M$ such that homomorphism $\pi_{1}\left(B_{S}\right) \rightarrow \pi_{1}(M)=\Gamma$ induced by inclusion is injective. The lower boundary $\partial_{-} B_{S}$ is a finite disjoint union of surfaces, which is nonempty since $\Gamma$ is not free. We let $\Gamma_{S}^{1}, \ldots, \Gamma_{S}^{k}<\Gamma$ denote the fundamental groups of the components- these groups inject into $\pi_{1}\left(B_{S}\right)$ and so also into $\Gamma$. Let $N_{S}^{i}=\mathbf{H}^{3} / \Gamma_{S}^{i} \rightarrow N$ be the associated covers.

If $S$ is incompressible, then $B_{S} \cong S \times[0,1], \partial_{-} B_{S}$ has one component and the cover $N_{S}^{1} \rightarrow N$ is the cover corresponding to $\pi_{1}(S)<\Gamma$. Since $\Gamma$ is not virtually a surface group, this is an infinite sheeted cover. By Theorem 2.1, $N_{S}^{1}$ has at least one geometrically finite end (in fact, it has exactly one geometrically finite end, and one simply degenerate end with a neighborhood that maps isometrically onto a neighborhood of $E$ in $N$ ). If $S$ is compressible, then $\Gamma_{S}^{i}$ has infinite index in $\pi_{1}\left(B_{S}\right)$, and hence also in $\Gamma$. Appealing to Theorem 2.1 again we see that $N_{S}^{i}$ has at least one geometrically finite end for each $1 \leq i \leq k$.

For each $i$, we obtain one or two pleated surfaces in $N$ as the image of $\partial C_{N_{S}^{i}}$ by the covering map $N_{S}^{i} \rightarrow N$. Let $\mathcal{P}_{E}$ denote the set of all such, as $i$ ranges from 1 to $k$. 
If $E_{1}, \ldots, E_{n}$ are the ends of $N$, then set

and put

$$
\mathcal{P}_{N}=\left\{\mathcal{P}_{E_{1}}, \ldots, \mathcal{P}_{E_{n}}\right\}
$$

$$
\mathcal{P}_{\Gamma}=q^{-1}\left(\mathcal{P}_{N}\right) \text {. }
$$

With this construction of pleated surfaces, together with the observation that the behaviour of the Bonahon compression bodies is natural with respect to finite coverings, it is straightforward to check that (1) and (2) hold. The remainder of the proof now proceeds as in case of $\Omega_{\Gamma} \neq \varnothing$, proving that $C(\Gamma)$ is discrete.

To prove the final statement that $[C(\Gamma): \Gamma]<\infty$, let $\mathcal{P}$ be the union of all the pleated surfaces in $\mathcal{P}_{\Gamma}$. Then

$$
[C(\Gamma): \Gamma]=\frac{\operatorname{Area}(\mathcal{P} / \Gamma)}{\operatorname{Area}(\mathcal{P} / C(\Gamma))}<\infty .
$$

This completes the proof.

\subsection{The surface group case}

Given Theorem 1.1 and Theorem 3.1 the proof of Theorem 1.2 will be completed upon establishing:

Theorem 3.2 Let $S$ be a closed orientable surface, $\rho: \pi_{1}(S) \rightarrow \operatorname{PSL}(2$, C) a faithful discrete representation and let $\Gamma=\rho\left(\pi_{1}(S)\right)$. Assume that $\Gamma$ is doubly degenerate.

Then $C(\Gamma)$ is discrete and finitely generated. Furthermore, $[C(\Gamma): \Gamma]=\infty$ if and only if $\Gamma$ is a fibre group. In this case, $C(\Gamma)$ is a lattice.

Before proving Theorem 3.2 we recall some terminology that will be used.

Definition Let $\Gamma$ be a Kleinian group. A point $x \in \Lambda_{\Gamma}$ is called a conical limit point of $\Gamma$ if for some (and hence every) geodesic ray $\tau$ in $\mathbf{H}^{3}$ ending at $x$, there is a compact set $K \subset \mathbf{H}^{3}$ such that $\{\gamma \in \Gamma: \gamma(\tau) \cap K \neq \varnothing\}$ is infinite.

We will make use of the following equivalent version of a conical limit point. We sketch a proof for convenience.

Lemma 3.3 Let $\Gamma$ be as above. The limit point $x$ is a conical limit point for $\Gamma$ if and only if $\Gamma$ contains a sequence of elements $\left\{\gamma_{m}\right\}$ such that the following conditions hold:

(i) $\gamma_{m}(x) \rightarrow x_{\infty}^{+} \in S_{\infty}^{2}$, and

(ii) $\gamma_{m} \mid S_{\infty}^{2} \backslash\{x\}$ converges uniformly on compact sets to a constant map with value $x_{\infty}^{-} \neq x_{\infty}^{+}$. 
Proof (Sketch) Let $x$ be a conical limit point and $\tau$ a geodesic ray in $\mathbf{H}^{3}$ ending at $x$. Because $x$ is conical, there exists infinitely many distinct elements $\left\{\gamma_{m}\right\} \subset \Gamma$ and compact set $K \subset \mathbf{H}^{3}$ such that $\gamma_{m}(\tau) \cap K \neq \varnothing$ for all $m$. Passing to a subsequence if necessary, it follows that $\gamma_{m}(\tau)$ limits to an oriented geodesic $\tau_{\infty}$ as $m \rightarrow \infty$. The positive and negative endpoints $x_{\infty}^{+}$and $x_{\infty}^{-}$, respectively, of $\tau_{\infty}$ clearly satisfy the conditions of the lemma with respect to the (sub)sequence $\left\{\gamma_{m}\right\} \subset \Gamma$.

Conversely, suppose $\left\{\gamma_{m}\right\} \subset \Gamma$ and $x_{\infty}^{ \pm}$are as in the lemma and let $\tau_{\infty}$ denote the oriented geodesic from $x_{\infty}^{-}$to $x_{\infty}^{+}$. Suppose $\tau$ is a geodesic ray ending at $x$. It is immediate that $\gamma_{m}(\tau)$ limits to the geodesic $\tau_{\infty}$ as $m \rightarrow \infty$. Letting $K$ be a closed ball in $\mathbf{H}^{3}$ of radius 1 , say, centered at some point of $\tau_{\infty}$. Then $\gamma_{m}(\tau)$ nontrivially intersects $K$ for all $m$ sufficiently large, and hence $x$ is a conical limit point.

We now commence with the proof of Theorem 3.2.

Proof of Theorem 3.2 Let $N=\mathbf{H}^{3} / \Gamma$ with ending laminations $v_{+}$and $v_{-}$. As these are distinct filling laminations, $v_{+}$and $v_{-}$have the property that they bind in the following sense (see Minsky [27, Lemma 2.4] and Kerckhoff [24], although in these papers, this property is called "filling" which we wish to avoid having used it earlier):

- The complement $S \backslash\left(v_{+} \cup v_{-}\right)$is a union of disks.

- Each such disc has boundary a finite collection of compact arcs coming alternately from $v_{+}$and $v_{-}$.

- All but a finite number of these disks have four sides.

We denote by $D_{1}, \ldots, D_{m}$ those finite number of disks which do not have four sides. Fix one of these disks $D$, which is contained in the intersection of two principal regions $P_{+}$of $v_{+}$and $P_{-}$of $v_{-}$. Let $\widetilde{D}$ denote some lift of $D$ to the universal cover $\widetilde{S}$ of $S$. Associated to $\widetilde{D}$ is a unique pair of lifts $\widetilde{P}_{+}$and $\widetilde{P}_{-}$of $P_{+}$and $P_{-}$, respectively, for which $\widetilde{D}=\widetilde{P}_{+} \cap \widetilde{P}_{-}$.

Let $\pi$ denote the restriction of the Cannon-Thurston map $\hat{\imath}$ to $S_{\infty}^{1}$. Theorem 2.2 shows that the map $\pi$ collapses $\partial_{\infty} \widetilde{P}_{+}$and $\partial_{\infty} \widetilde{P}_{-}$to points $x_{+}$and $x_{-}$in $\Lambda_{\Gamma}=S_{\infty}^{2}$. Since the laminations bind, the closure of $\widetilde{D}$ is compact and therefore $\partial_{\infty} \widetilde{P}_{+} \cap \partial_{\infty} \widetilde{P}_{-}=\varnothing$. It follows that $x_{+}$and $x_{-}$are distinct points in $S_{\infty}^{2}$. We call these points special points.

Doing this for each of $D_{1}, \ldots, D_{m}$ determines a finite number of pairs of special points

$$
\left(x_{+}^{(1)}, x_{-}^{(1)}\right),\left(x_{+}^{(2)}, x_{-}^{(2)}\right), \ldots,\left(x_{+}^{(m)}, x_{-}^{(m)}\right)
$$

in $S_{\infty}^{2}$ 
Connect $x_{+}^{(j)}$ and $x_{-}^{(j)}$ by a geodesic $\xi^{(j)}$ in $\mathbf{H}^{3}$ for each $j=1, \ldots, m$ and let $\mathcal{X}(\Gamma)$ be the $\Gamma$-orbit of this collection of geodesics. We shall refer to a geodesic in $\mathcal{X}(\Gamma)$ as a special geodesic. We record the following observation that is crucial in what follows.

Theorem 3.4 Let $x \in S_{\infty}^{2}$ have the property that $\left|\pi^{-1}(x)\right|>1$. Then $x$ is not a conical limit point. In particular, for each $i=1,2, \ldots, m, x_{+}^{(i)}$ and $x_{-}^{(i)}$ are not conical limit points.

Proof Given $x$ as in the hypothesis of Theorem 3.4, Theorem 2.2 shows that there are $a_{1}, \ldots, a_{k} \in S_{\infty}^{1}(k \geq 2)$, which are endpoints of a leaf or ideal vertices of a principal region of $\widetilde{v}_{+}$or $\widetilde{v}_{-}$and which satisfy

$$
x=\pi\left(a_{1}\right)=\cdots=\pi\left(a_{k}\right) .
$$

Moreover, by Theorem 2.2, these are the only identifications. We also recall that by definition of a Cannon-Thurston map, $\pi$ is equivariant with respect to $\rho$ via the action of $\pi_{1}(S)$ on $S^{1}=S_{\infty}^{1}$ and $\Gamma$ on $S^{2}=S_{\infty}^{2}$.

We now argue by contradiction, and assume that $x$ is a conical limit point. Lemma 3.3 provides a sequence of distinct elements $\left\{\gamma_{n}\right\}_{n=1}^{\infty} \subset \Gamma$ so that

(1) $\gamma_{n}(x) \rightarrow x_{\infty}^{+} \in S^{2}$ as $n \rightarrow \infty$, and

(2) $\gamma_{n} \mid S^{2}-\{x\}$ converges uniformly on compact sets to a constant map with value $x_{\infty}^{-} \neq x_{\infty}^{+}$.

Let $\left\{g_{n}\right\}_{n=1}^{\infty} \subset \pi_{1}(S)$ be such that $\rho\left(g_{n}\right)=\gamma_{n}$, and pass to a subsequence if necessary so that as $n \rightarrow \infty, g_{n}\left(a_{1}\right) \rightarrow a_{\infty} \in S^{1}$ for some $a_{\infty} \in S^{1}$. Since $\pi$ is continuous and equivariant with respect to $\rho$, we have

$$
\pi\left(a_{\infty}\right)=\lim _{n \rightarrow \infty} \pi\left(g_{n}\left(a_{1}\right)\right)=\lim _{n \rightarrow \infty} \rho\left(g_{n}\right)\left(\pi\left(a_{1}\right)\right)=\lim _{n \rightarrow \infty} \gamma_{n}(x)=x_{\infty}^{+} .
$$

Next, let $b, c \in S^{1} \backslash\left\{a_{1}, \ldots, a_{k}\right\}$ be distinct points bounding an interval $I \subset S^{1}$ containing $a_{2}, \ldots, a_{k}$ and not containing $a_{1}$. (See Figure 1.) Pass to a further subsequence if necessary so that as $n \rightarrow \infty, g_{n}(b) \rightarrow b_{\infty}$ and $g_{n}(c) \rightarrow c_{\infty}$ for some points $b_{\infty}, c_{\infty} \in S^{1}$.

Appealing to continuity and equivariance of $\pi$ again we have

$$
\begin{aligned}
& \pi\left(b_{\infty}\right)=\lim _{n \rightarrow \infty} \pi\left(g_{n}(b)\right)=\lim _{n \rightarrow \infty} \gamma_{n}(\pi(b))=x_{\infty}^{-} \neq x_{\infty}^{+}=\pi\left(a_{\infty}\right), \\
& \pi\left(c_{\infty}\right)=\lim _{n \rightarrow \infty} \pi\left(g_{n}(c)\right)=\lim _{n \rightarrow \infty} \gamma_{n}(\pi(c))=x_{\infty}^{-} \neq x_{\infty}^{+}=\pi\left(a_{\infty}\right) .
\end{aligned}
$$




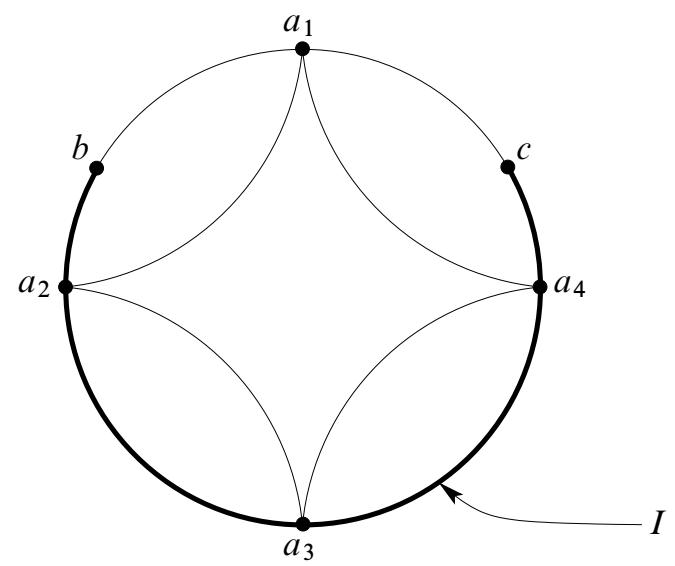

Figure 1

Since the action of $\pi_{1}(S)$ on $\widetilde{S} \cup S^{1}$ is Fuchsian, a well-known characterization of Fuchsian groups (see Scott [36, page 484]) now allows us to deduce that $\pi_{1}(S)$ acts properly discontinuously on the space $\left(S^{1} \times S^{1} \times S^{1}\right) \backslash \Delta$ where

$$
\Delta=\left\{(p, q, r) \in S^{1} \times S^{1} \times S^{1}: \text { two of } p, q, r \text { are equal }\right\} .
$$

Hence $\left(a_{\infty}, b_{\infty}, c_{\infty}\right)$ cannot be a triple of distinct points in $S^{1}$ since it is the limit of $\left\{g_{n}\left(a_{1}, b, c\right)\right\}_{n=1}^{\infty}$. From (3) and (4), $a_{\infty} \neq b_{\infty}$ and $a_{\infty} \neq c_{\infty}$. Therefore, $b_{\infty}=c_{\infty}$. It follows that after passing to another subsequence if necessary, $\left\{g_{n}\right\}$ restricted to one of the intervals bounded by $b$ and $c$, call it $J$, must converge to a constant map with value $b_{\infty}=c_{\infty}$ (this is because each $g_{n}$ is a homeomorphism, and the images of the endpoints are converging to the same point). We cannot have $a_{1} \in J$ since $g_{n}\left(a_{1}\right) \rightarrow a_{\infty} \neq b_{\infty}$ as $n \rightarrow \infty$. Therefore, $J=I$, which is to say, $g_{n}$ restricted to $I$ converges to the constant $b_{\infty}$. However, this interval contains $a_{2}$, and so $g_{n}\left(a_{2}\right) \rightarrow b_{\infty}$ as $n \rightarrow \infty$. Another application of continuity and equivariance of $\pi$ tells us

$$
\pi\left(b_{\infty}\right)=\lim _{n \rightarrow \infty} \pi\left(g_{n}\left(a_{2}\right)\right)=\lim _{n \rightarrow \infty} \gamma_{n}\left(\pi\left(a_{2}\right)\right)=\lim _{n \rightarrow \infty} \gamma_{n}(x)=x_{\infty}^{+},
$$

which is a contradiction to (3).

Remark It is classical (see Beardon and Maskit [3]) that a finitely generated Kleinian group without parabolic elements is geometrically finite if and only if all limit points are conical limit points. It is interesting that properties of the Cannon-Thurston map apply to produce a natural class of "explicit" nonconical limit points for doubly degenerate surface groups.

The key claims needed for the proof of Theorem 3.2 are now contained in the following proposition. 
Proposition $3.5 \quad(1) \mathcal{X}(\Gamma)$ is a locally finite collection of geodesics in $\mathbf{H}^{3}$.

(2) If $\Gamma_{1}$ is a subgroup of finite index in $\Gamma$ then $\mathcal{X}\left(\Gamma_{1}\right)=\mathcal{X}(\Gamma)$.

(3) If $g \in \operatorname{PSL}(2, \mathbf{C})$, then $\mathcal{X}\left(g \Gamma g^{-1}\right)=g \mathcal{X}(\Gamma)$.

Proof To prove (1), we suppose to the contrary that there exists a compact set $K \subset \mathbf{H}^{3}$ that intersects infinitely many geodesics in $\mathcal{X}(\Gamma)$ and arrive at a contradiction. As $\mathcal{X}(\Gamma)$ is the orbit of finitely many geodesics $\left\{\xi^{(1)}, \ldots, \xi^{(m)}\right\}$, there exists $1 \leq j \leq m$ and a sequence of distinct elements $\left\{\gamma_{n}\right\}_{n=1}^{\infty} \subset \Gamma$ so that $K \cap \gamma_{n}\left(\xi^{(j)}\right) \neq \varnothing$ for all $n$. Equivalently, $\gamma_{n}^{-1}(K) \cap \xi^{(j)} \neq \varnothing$ for all $n$. However, $\xi^{(j)}$ joins $x_{+}^{(j)}$ and $x_{-}^{(j)}$ and so by definition at least one of these points is a conical limit point. This contradicts Theorem 3.4, and hence part (1) holds.

For part (2), we let $\Gamma_{1}<\Gamma$ be a finite index subgroup and $p: S_{1} \rightarrow S$ the associated finite sheeted cover. As we observed earlier, it follows from its well-definition that the ending laminations for $\Gamma_{1}$ are the preimage $p^{-1}\left(v_{ \pm}\right)$of the ending laminations for $\Gamma$. Since the universal coverings $q: \widetilde{S} \rightarrow S$ and $q_{1}: \widetilde{S} \rightarrow S_{1}$ satisfy $q=p \circ q_{1}$, it follows that $v_{ \pm}$and $p^{-1}\left(v_{ \pm}\right)$define the same set of principal regions in $\widetilde{S}$. Since $\mathcal{X}\left(\Gamma_{1}\right)$ and $\mathcal{X}(\Gamma)$ are defined in terms of these regions, properties of the Cannon-Thurston map described in Theorem 2.2 imply $\mathcal{X}\left(\Gamma_{1}\right)=\mathcal{X}(\Gamma)$.

To prove (3), we note that for a fixed $g \in \operatorname{PSL}(2, \mathbf{C})$, the equivariant map associated to the conjugated degenerate group $g \rho\left(\pi_{1}(S)\right) g^{-1}$ is obtained as a composition $g \circ \iota: \widetilde{S} \longrightarrow \mathbf{H}^{3}$. Indeed, for all $\gamma \in \pi_{1}(S)$, the action of $\gamma$ on $\widetilde{S}$ and on $\mathbf{H}^{3}$ via $\rho$ and its conjugate fit into the following commutative diagram:

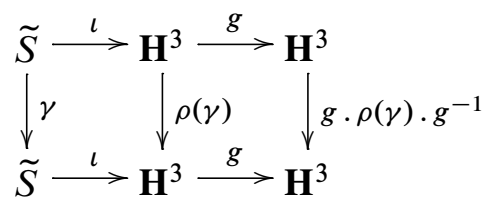

Now $g$ is a hyperbolic isometry and therefore admits an extension to $\mathbf{H}^{3} \cup S_{\infty}^{2}$ and one sees that the two maps

$$
\widehat{g \circ \iota}, g \circ \widehat{\iota}: \widetilde{S} \cup S_{\infty}^{1} \longrightarrow \mathbf{H}^{3} \cup S_{\infty}^{2}
$$

agree on the dense set $\widetilde{S}$, hence everywhere by continuity. Since the geodesics in $\mathcal{X}$ are defined in terms of the boundary values of the Cannon-Thurston map, the result follows.

The proof of the first part of Theorem 3.2 will follow from the next claim. For if $C(\Gamma)$ is not discrete we can find a collection of geodesics in $\mathcal{X}(\Gamma)$ violating (1) of Proposition 3.5. 
Claim 1 Suppose that $g \in C(\Gamma)$, then $g$ leaves $\mathcal{X}(\Gamma)$ setwise invariant.

Proof of Claim 1 Let $g \in C(\Gamma)$. Then $\Gamma \cap g \Gamma g^{-1}$ has finite index in $\Gamma$ and $g \Gamma g^{-1}$. Proposition 3.5 (2) shows that the collection of special geodesics associated to $\Gamma \cap g \Gamma g^{-1}$ is $\mathcal{X}(\Gamma)$, and Proposition 3.5 (3) shows that $g(\mathcal{X}(\Gamma))$ is the set of special geodesics associated to $g \Gamma g^{-1}$. Proposition 3.5 (2) applied to $\Gamma \cap g \Gamma g^{-1}$ as a subgroup of $g \Gamma g^{-1}$ now completes the proof of the claim.

We now prove:

Theorem 3.6 $C(\Gamma)$ is finitely generated.

Proof For $\xi \in \mathcal{X}(\Gamma)$, set $\operatorname{Stab}(\xi)=\{g \in C(\Gamma): g \xi=\xi\}$. Since $C(\Gamma)$ is discrete, it follows that each $\operatorname{Stab}(\xi)$ is either a finite cyclic or dihedral group, or an extension of an infinite cyclic or infinite dihedral group by a finite cyclic group. In any case, $\operatorname{Stab}(\xi)$ is finitely generated.

Now recall that $\mathcal{X}(\Gamma)$ is the $\Gamma$ orbit of the finite set of geodesics $\left\{\xi^{(1)}, \ldots, \xi^{(m)}\right\}$. Let $\mathcal{D}_{r}=\operatorname{Stab}\left(\xi^{(r)}\right)$ for each $r=1, \ldots, m$. Then for any $\xi \in \mathcal{X}(\Gamma)$, there exists $\gamma \in \Gamma$ and $1 \leq r \leq m$ such that $\xi=\gamma\left(\xi^{(r)}\right)$ and so

$$
\operatorname{Stab}(\xi)=\gamma \operatorname{Stab}\left(\xi^{(r)}\right) \gamma^{-1}=\gamma \mathcal{D}_{r} \gamma^{-1}
$$

Let $K_{1}=\left\langle\mathcal{D}_{1}, \ldots, \mathcal{D}_{m}, \Gamma\right\rangle$, and consider the action of $K_{1}$ on $\mathcal{X}(\Gamma)$. Since $\Gamma<K_{1}$, this has at most $m$ orbits. Suppose that there is an element $\alpha_{1} \in C(\Gamma)$ which identifies some of the orbits not identified by $K_{1}$. Set $K_{2}=\left\langle\alpha_{1}, K_{1}\right\rangle$. We repeat this process: since the number of orbits can only go down, we may continue in this way until reaching $K_{j}$, a subgroup for which there are no extra orbit identifications (beyond those arising from $K_{j}$ ) possible for any choice of an element of $C(\Gamma)$. The proof of the theorem is completed by the next Claim.

Claim 2 In this situation, $K_{j}=C(\Gamma)$.

Proof of Claim 2 Pick $\beta \in C(\Gamma)$ and choose any geodesic $\xi \in \mathcal{X}(\Gamma)$. Then $\beta(\xi)=$ $k(\xi)$ for some $k \in K_{j}$ so that $k^{-1} \cdot \beta$ stabilises $\xi$, and therefore lies in $\gamma \cdot \mathcal{D}_{r} \cdot \gamma^{-1}$ for some $\gamma \in \Gamma$ and one of the stabiliser groups $\mathcal{D}_{r}$. Since $\gamma \cdot \mathcal{D}_{r} \cdot \gamma^{-1} \leq K_{j}$, it follows that $\beta \in K_{j}$ so proving the claim and completing the proof of the theorem.

Remark Theorem 3.6 follows from the next theorem, but it seemed interesting to give a direct proof using the structure of the special geodesics. 
We now determine when $[C(\Gamma): \Gamma]$ is finite, completing the proof of Theorem 3.2.

Theorem 3.7 The group $\Gamma$ is a fibre group if and only if $[C(\Gamma): \Gamma]=\infty$.

Proof Let $\Gamma_{0}<\Gamma$ be the largest surface subgroup (which has index at most 2 inside $\Gamma$ ). If $\Gamma$ is a fibre group then $[C(\Gamma): \Gamma]$ is infinite. Indeed, $C\left(\Gamma_{0}\right)=C(\Gamma)$ contains $N_{\mathrm{PSL}(2, \mathbf{C})}\left(\Gamma_{0}\right)$ (the normalizer of $\Gamma_{0}$ in $\operatorname{PSL}(2, \mathbf{C})$ ), which is a lattice, and we deduce that $C(\Gamma)$ is a lattice.

Now suppose $[C(\Gamma): \Gamma]=\infty$, and consider the infinite sheeted covering

$$
p: \mathbf{H}^{3} / \Gamma \longrightarrow \mathbf{H}^{3} / C(\Gamma) .
$$

The manifold $\mathbf{H}^{3} / \Gamma$ has at most two ends which are (both) geometrically infinite by assumption, and so $p$ is infinite-to-one on a neighborhood of at least one of these ends. It follows from Theorem 2.1, that $\Gamma$ is a fibre group.

\section{Parabolics}

Here we explain the mild generalization of Theorem 1.2 where we allow our groups to contain parabolics.

Theorem 4.1 Suppose $\Gamma<\operatorname{PSL}(2, \mathbf{C})$ is a finitely generated torsion free Kleinian group of the first kind which is not a lattice. If $\Gamma$ is not free and is not the fundamental group of a compression body with toroidal lower boundary, then $C(\Gamma)$ is discrete. Moreover, $[C(\Gamma): \Gamma]=\infty$ if and only if $\Gamma$ is a fiber group.

Proof (Sketch) If $\Gamma$ has no parabolics, then this reduces to Theorem 1.2, so we assume that $\Gamma$ contains parabolic elements.

We refer the reader to Canary $[10 ; 11]$ for terminology and a detailed discussion of the notation used here. Let $q: \mathbf{H}^{3} \rightarrow N=\mathbf{H}^{3} / \Gamma$ be the universal covering, $\epsilon>0$ some number less than the 3-dimensional Margulis constant, and let $N_{\epsilon}^{0}$ denote the result of removing the cuspidal $\epsilon$-thin part of $N$; the Tameness Theorem implies $N_{\epsilon}^{0}$ is tame [1; 9]. Let $M \subset N_{\epsilon}^{0}$ denote a relative compact core for $N_{\epsilon}^{0}$ : a compact core for which $P=M \cap \partial N_{\epsilon}^{0}$ is a disjoint union of annuli and tori in $\partial M$ and so that the ends of $N_{\epsilon}^{0}$ are in a one-to-one correspondence with the components of $\partial M-P$.

For any group $\Gamma$ as in the statement of the theorem, we need to find a finite set $\mathcal{P}_{N}$ of finite area pleated surfaces $\mathcal{P}_{N}$ in $N$, such that the locally finite collection of pleated surfaces

$$
\mathcal{P}_{\Gamma}=q^{-1}\left(\mathcal{P}_{N}\right)
$$

satisfies the following two properties (cf (2) and (1) from Section 3.1). 
(1) For any finite index subgroup $\widehat{\Gamma}<\Gamma$ we have $\mathcal{P}_{\widehat{\Gamma}}=\mathcal{P}_{\Gamma}$,

(2) For all $g \in \operatorname{PSL}(2, \mathbf{C})$, we have $\mathcal{P}_{g \Gamma g^{-1}}=g\left(\mathcal{P}_{\Gamma}\right)$.

Given these two properties, the proof of the Theorem follows as in the proof of Theorem 3.1.

First, suppose that $\Gamma$ is not virtually a surface group and fix a nontoroidal component $S \subset \partial M$, which exists since $\Gamma$ is not a lattice. Let $B_{S}$ be the compression body associated to $S$ from [5] as in the proof of Theorem 3.1. By hypothesis, $B_{S}$ is not a handlebody and so has some nontrivial lower boundary $\partial_{-} B_{S} \neq \varnothing$. Furthermore, we claim that $\partial_{-} B_{S}$ cannot be a union of tori. To see this, note that this would imply that either we could take $B_{S}=M$, or else some component $T \subset \partial_{-} B_{S}$ is not peripheral in $M$. The former case is ruled out by the hypothesis that $\Gamma=\pi_{1}(M)$ is not the fundamental group of a compression body with toroidal lower boundary, while the latter case is impossible since $M$ is the core of a hyperbolic manifold, so has no nonperipheral incompressible tori.

Let $\Sigma_{1}, \ldots, \Sigma_{k} \subset \partial_{-} B_{S}$ denote the set of nontoroidal components (which is nonempty) and let $\Gamma(1, S), \ldots, \Gamma(k, S)<\Gamma$ be the associated nonempty, finite collection of surface subgroups. Since we have assumed that $\Gamma$ is not virtually a surface group, we have $[\Gamma: \Gamma(j, S)]=\infty$ for all $1 \leq j \leq k$.

For each $1 \leq j \leq k$, let $N(j, S)=\mathbf{H}^{3} / \Gamma(j, S)$ and $N_{\epsilon}^{0}(j, S)$ the complement of the cuspidal $\epsilon$-thin part in $N(j, S)$. Appealing to the covering theorem (for 3manifolds with cusps; see $[11 ; 13])$, we again deduce that at least one end of $N_{\epsilon}^{0}(j, S)$ is geometrically finite. The boundary of the convex core $C_{\Gamma(j, S)}$ maps into $N$, and the set of all such surfaces is a finite collection of finite area hyperbolic surfaces canonically associated to $S$. Let $\mathcal{P}_{N}$ be the set of these pleated surfaces over all component $S \subset \partial M$. Properties (1) and (2) are easily verified for this family, and discreteness of $C(\Gamma)$ follows.

If $\Gamma$ is a virtually a surface group, then since we have assumed $\Gamma$ has parabolics, $P \subset \partial M$ is a nonempty collection of annuli. For simplicity, assume $M=S \times[0,1]$ (if $M$ is a twisted $I$-bundle, take the two-fold cover). Let $\Sigma \subset \partial M-P$ be a component whose closure nontrivially meets $P$ and let $\Gamma_{\Sigma} \subset \Gamma$ denote the (injective) image of $\pi_{1}(\Sigma)$ in $\pi_{1}(S)=\Gamma$. There is at least one such $\Sigma$ since $P \neq \varnothing$. Since $\Sigma$ corresponds to a proper subsurface of $S, \Gamma_{\Sigma}$ has infinite index in $\Gamma$. Letting $N(\Sigma)=\mathbf{H}^{3} / \Gamma_{\Sigma}$ and $N_{\epsilon}^{0}(\Sigma)$ be the complement of the $\epsilon$-thin cuspidal part, arguing as above one sees that $N_{\epsilon}^{0}(\Sigma)$ has at least one geometrically finite end. Therefore, we can associate to $\Sigma$, the components of $C_{\Gamma_{\Sigma}}$ mapped into $N$, which is a finite 
set of finite area pleated surfaces. The set of all of these taken over all components $\Sigma \subset \partial M-P$ as above gives the required set of pleated surfaces.

Setting $\mathcal{P}$ to be the union of all the surfaces in $\mathcal{P}_{\Gamma}$ we have

$$
[C(\Gamma): \Gamma]=\frac{\operatorname{Area}(\mathcal{P} / \Gamma)}{\operatorname{Area}(\mathcal{P} / C(\Gamma))} .
$$

It follows that under the hypotheses of the theorem, if $\Gamma$ has parabolics, $[C(\Gamma): \Gamma]<\infty$. If $\Gamma$ has no parabolics, then applying Theorem 1.2 completes the proof.

\section{References}

[1] I Agol, Tameness of hyperbolic 3-manifolds arXiv:math/0405568

[2] L Bartholdi, O Bogopolski, On abstract commensurators of groups arXiv: 0902.4542

[3] A F Beardon, B Maskit, Limit points of Kleinian groups and finite sided fundamental polyhedra, Acta Math. 132 (1974) 1-12 MR0333164

[4] J A Behrstock, W D Neumann, Quasi-isometric classification of graph manifold groups, Duke Math. J. 141 (2008) 217-240 MR2376814

[5] F Bonahon, Cobordism of automorphisms of surfaces, Ann. Sci. École Norm. Sup. (4) 16 (1983) 237-270 MR732345

[6] F Bonahon, Bouts des variétés hyperboliques de dimension 3, Ann. of Math. (2) 124 (1986) 71-158 MR847953

[7] J R Brock, R D Canary, Y N Minsky, The classification of Kleinian surface groups II: the ending lamination conjecture arXiv:math/0412006

[8] J R Brock, R D Canary, Y N Minsky, The classification of finitely generated Kleinian groups, in preparation

[9] D Calegari, D Gabai, Shrinkwrapping and the taming of hyperbolic 3-manifolds, J. Amer. Math. Soc. 19 (2006) 385-446 MR2188131

[10] R D Canary, Ends of hyperbolic 3-manifolds, J. Amer. Math. Soc. 6 (1993) 1-35 MR1166330

[11] R D Canary, A covering theorem for hyperbolic 3-manifolds and its applications, Topology 35 (1996) 751-778 MR1396777

[12] R D Canary, Marden's tameness conjecture: history and applications, from: "Geometry, analysis and topology of discrete groups", (L Ji, K Liu, L Yang, S-T Yau, editors), Adv. Lect. Math. 6, Int. Press, Somerville, MA (2008) 137-162 MR2464394

[13] R D Canary, C J Leininger, Kleinian groups with discrete length spectrum, Bull. Lond. Math. Soc. 39 (2007) 189-193 MR2323447 
[14] J W Cannon, W P Thurston, Group invariant Peano curves, Geom. Topol. 11 (2007) 1315-1355 MR2326947

[15] A J Casson, S A Bleiler, Automorphisms of surfaces after Nielsen and Thurston, London Math. Society Student Texts 9, Cambridge Univ. Press (1988) MR964685

[16] D B A Epstein, A Marden, Convex hulls in hyperbolic space, a theorem of Sullivan, and measured pleated surfaces, from: "Fundamentals of hyperbolic geometry: selected expositions", (R D Canary, D B A Epstein, A Marden, editors), London Math. Soc. Lecture Note Ser. 328, Cambridge Univ. Press (2006) 117-266 MR2235711

[17] B Farb, S Weinberger, Hidden symmetries and arithmetic manifolds, from: "Geometry, spectral theory, groups, and dynamics”, (M Entov, Y Pinchover, M Sageev, editors), Contemp. Math. 387, Amer. Math. Soc. (2005) 111-119 MR2179789

[18] B Farb, S Weinberger, Isometries, rigidity and universal covers, Ann. of Math. (2) 168 (2008) 915-940 MR2456886

[19] O Goodman, D Heard, C Hodgson, Commensurators of cusped hyperbolic manifolds, Experiment. Math. 17 (2008) 283-306 MR2455701

[20] L Greenberg, Commensurable groups of Moebius transformations, from: "Discontinuous groups and Riemann surfaces (Proc. Conf., Univ. Maryland, College Park, Md., 1973)", (L Greenberg, editor), Ann. of Math. Studies 79, Princeton Univ. Press (1974) 227-237 MR0379689

[21] L Greenberg, Finiteness theorems for Fuchsian and Kleinian groups, from: "Discrete groups and automorphic functions (Proc. Conf., Cambridge, 1975)", (W J Harvey, editor), Academic Press, London (1977) 199-257 MR0585138

[22] J Hempel, 3-Manifolds, Ann. of Math. Studies 86, Princeton Univ. Press (1976) MR0415619

[23] M Kapovich, On the absence of Sullivan's cusp finiteness theorem in higher dimensions, from: "Algebra and analysis (Irkutsk, 1989)", (L A Bokut', M Hazewinkel, Y G Reshetnyak, editors), Amer. Math. Soc. Transl. Ser. 2 163, Amer. Math. Soc. (1995) 77-89 MR1331386

[24] S P Kerckhoff, Lines of minima in Teichmüller space, Duke Math. J. 65 (1992) 187-213 MR1150583

[25] C J Leininger, D Margalit, Abstract commensurators of braid groups, J. Algebra 299 (2006) 447-455 MR2228321

[26] G A Margulis, Discrete subgroups of semisimple Lie groups, Ergebnisse der Math. und ihrer Grenzgebiete (3) 17, Springer-Verlag, Berlin (1991) MR1090825

[27] Y N Minsky, On rigidity, limit sets, and end invariants of hyperbolic 3-manifolds, J. Amer. Math. Soc. 7 (1994) 539-588 MR1257060

[28] Y N Minsky, The classification of Kleinian surface groups. I. Models and bounds, Ann. of Math. (2) 171 (2010) 1-107 MR2630036 
[29] M Mitra, Cannon-Thurston maps for hyperbolic group extensions, Topology 37 (1998) 527-538 MR1604882

[30] M Mj, Cannon-Thurston maps for surface groups arXiv:math/0607509

[31] M Mj, Ending laminations and Cannon-Thurston maps arXiv:math/0701725

[32] W D Neumann, A W Reid, Arithmetic of hyperbolic manifolds, from: “Topology '90 (Columbus, OH, 1990)", (B Apanasov, W D Neumann, A W Reid, L Siebenmann, editors), Ohio State Univ. Math. Res. Inst. Publ. 1, de Gruyter, Berlin (1992) 273-310 MR1184416

[33] A W Reid, GS Walsh, Commensurability classes of 2-bridge knot complements, Algebr. Geom. Topol. 8 (2008) 1031-1057 MR2443107

[34] R E Schwartz, The quasi-isometry classification of rank one lattices, Inst. Hautes Études Sci. Publ. Math. (1995) 133-168 (1996) MR1383215

[35] G P Scott, Compact submanifolds of 3-manifolds, J. London Math. Soc. (2) 7 (1973) 246-250 MR0326737

[36] P Scott, The geometries of 3-manifolds, Bull. London Math. Soc. 15 (1983) 401-487 MR705527

[37] Y Shalom, Rigidity of commensurators and irreducible lattices, Invent. Math. 141 (2000) 1-54 MR1767270

[38] G Shimura, Introduction to the arithmetic theory of automorphic functions, Kanô Memorial Lectures 1, Publ. of the Math. Soc. Japan 11, Iwanami Shoten, Publ., Tokyo; Princeton Univ. Press (1971) MR0314766

[39] W P Thurston, Hyperbolic structures on 3-manifolds II: Surface groups and 3manifolds which fiber over the circle arXiv:math.GT/9801045

[40] W P Thurston, The geometry and topology of three-manifolds, Princeton Univ. Math. Dept. Lecture Notes (1979) Available at http://msri.org/publications/books/ gt $3 \mathrm{~m} /$

Department of Mathematics, University of Illinois at Urbana-Champaign

Urbana IL 61801, USA

Department of Mathematics, University of California, Santa Barbara

Santa Barbara CA 93106, USA

Department of Mathematics, University of Texas

Austin TX 78712, USA

clein@math.uiuc.edu, long@math.ucsb.edu, areid@math.utexas.edu

Received: 27 July $2010 \quad$ Revised: 27 December 2010 